\title{
Dynamic organization of chromosomal DNA in Escherichia coli
}

\author{
Hironori Niki, ${ }^{1-3}$ Yoshiharu Yamaichi, ${ }^{2}$ and Sota Hiraga ${ }^{2}$

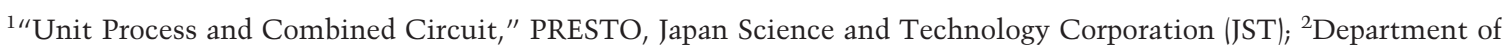 \\ Molecular Cell Biology, Institute of Molecular Embryology and Genetics, Kumamoto University School of Medicine, \\ Kumamoto, 862-0976, Japan
}

\begin{abstract}
We have revealed the subcellular localization of different DNA segments that are located at $\sim 230$-kb intervals on the Escherichia coli chromosome using fluorescence in situ hybridization (FISH). The series of chromosome segments is localized within the cell in the same order as the chromosome map. The large chromosome region including oriC shows similar localization patterns, which we call the Ori domain. In addition, the localization pattern of the large segment including dif is characteristic of the replication terminus region. The segment also shows similar localization patterns, which we call the Ter domain. In newborn cells, Ori and Ter domains of the chromosome are differentially localized near opposite cell poles. Subsequently, in the B period, the Ori domain moves toward mid-cell before the initiation of replication, and the Ter domain tends to relocate at mid-cell. An inversion mutant, in which the Ter domain is located close to oriC, shows abnormal subcellular localization of ori and dif segments, resulting in frequent production of anucleate cells. These studies thus suggest that the $E$. coli chromosome is organized to form a compacted ring structure with the Ori and Ter domains; these domains participate in the cell cycle-dependent localization of the chromosome.
\end{abstract}

[Key Words: Prokaryote; cell division; FISH; nucleoid; partitioning; segregation]

Received October 18, 1999; revised version accepted December 8, 1999.

The bacterial chromosome, 1000 times the length of the bacterium, is folded by an unknown mechanism and organized in a compact form called the nucleoid. Carefully isolated nucleoids from Escherichia coli have been investigated in vitro (for review, see Pettijohn 1996). The nucleoid is a closed duplex structure with a series of loops. The DNA loops are negatively supercoiled, resulting in further compaction. Moreover, several DNA-binding proteins, including $\mathrm{HU}, \mathrm{IHF}$, and $\mathrm{H}-\mathrm{NS}$, may promote a compact state of DNA in nucleoids. Interestingly, mutants defective in HU and/or H-NS accumulate anucleate cells (Wada et al. 1988; Kaidow et al. 1995). The compaction of nucleoids may be related to the mechanism of chromosome partitioning. This possibility is strongly supported by investigations of bacterial homologs of the eukaryotic SMC (structural maintenance of chromosome) protein. The SMC protein is involved in chromosome condensation, pairing, and partitioning in eukaryotic cells from yeast to human (for review, see Hirano 1999). In Bacillus subtilis, smc null mutations cause a temperature-sensitive lethal phenotype and failure to partition the chromosome (Britton et al. 1998; Moriya et al. 1998), similar to $m u k B$ null mutants in E. coli (Niki et al. 1991). The MukB protein does

${ }^{3}$ Corresponding author.

E-MAIL niki@gpo.kumamoto-u.ac.jp; FAX 81-96-371-2408. not share the common amino acid motif found in the SMC family. Nevertheless, the morphological structure of the MukB protein molecules is remarkably similar to that of SMC proteins (Niki et al. 1992; Melby et al. 1998). Thus, elucidation of the structure of compacted nucleoids and chromosome organization in vivo is important for understanding the mechanism of chromosome partitioning.

The bacterial nucleoid is amorphous in vivo because the folded chromosome is not highly condensed throughout the cell division cycle. Replication and transcription take place simultaneously, even during chromosome partitioning. The DNA packing density in the nucleoid has been estimated in exponentially grown $E$. coli cells (Kellenberger 1990). It is similar to that of interphase nuclei of eukaryotes. Therefore, whole chromosomes seem to migrate to daughter cells with coupling to cell elongation but not by an active mechanism such as a mitotic apparatus. Recently, it has become apparent that specific DNA segments on bacterial chromosomes migrate rapidly during chromosome partitioning (Glaser et al. 1997; Gordon et al. 1997; Lin et al. 1997; Webb et al. 1997, 1998; Niki and Hiraga 1998). The results revealed that the oriC DNA segment in newborn cells is localized at a nucleoid border and the replication terminus DNA segment is localized at the opposite nucleoid border. One copy of the replicated oriC segment remains at its nucleoid border and the other copy migrates to the opposite 
nucleoid border. On the other hand, the terminal DNA segment migrates from the nucleoid border to mid-cell in the early stage of the cell division cycle. Moreover, chromosomal DNA segments midway between oriC and the replication terminus tend to be localized at subcellular positions between oriC and the terminus (Teleman et al. 1998). Cytological methods, based on subcellular localization of a few chromosome segments, have thus revealed a dynamic organization of bacterial chromosomes. Further examination of various segments on the chromosome clearly reveals the organization of the whole nucleoid.

The cell division cycle of bacteria can be defined in terms of two constants, the $\mathrm{C}$ and $\mathrm{D}$ periods. During the E. coli cell division cycle, the $\mathrm{C}$ period is the time during which a round of chromosome replication takes place, lasting $40 \mathrm{~min}$ at $37^{\circ} \mathrm{C}$. The $\mathrm{D}$ period is the period between completion of chromosome replication and the subsequent cell division $-20 \mathrm{~min}$ at $37^{\circ} \mathrm{C}$. When cells duplicate every $60 \mathrm{~min}$ at $37^{\circ} \mathrm{C}$ /doubling time of $60 \mathrm{~min}$ ), chromosome replication initiates soon after the cells divide. Cells growing at doubling times of $>60$ min may have an additional stage, the B period, between birth of the newborn cell and the initiation of replication, which resembles the $G_{1}$ phase of eukaryotic cell cycle.

In the present work, we examine the subcellular localization of 22 DNA segments of the E. coli chromosome by the FISH method. The series of chromosome segments was localized within the cell in the same order as the chromosome map. Our results demonstrate that the circular chromosomal DNA is organized to form a compact ring structure in the cell. The dynamic organization of the nucleoid plays a critical role in chromosome replication, partitioning, and cell division in the bacterial cell cycle.

\section{Results \\ The oriC DNA segment is localized at midcell prior to initiation of replication in slowly growing cells}

oriC plasmids are not localized at specific subcellular sites and may be replicated anywhere in the cytoplasm (Niki and Hiraga 1999). However, some components of replication machinery are localized at or near mid-cell during chromosome replication (Lemon and Grossman 1998). To determine where oriC on the chromosome is localized in the cell at initiation of chromosome replication, we examined the localization of oriC in slowly growing cells at $37^{\circ} \mathrm{C}$.

Figure 1A shows the subcellular localization of the oriC segment in cultures growing at doubling times of 52,80 , and $280 \mathrm{~min}$. When the doubling time was 52 min, in cells with a single oriC focus, the focus was broadly distributed near the one-quarter position of cell length, but not at mid-cell (Fig. 1A, a). In the cells, 14.6\% of the oriC foci were localized at $40 \%-50 \%$ of cell length. This is consistent with our previous result, with cells growing at a doubling time of $55 \mathrm{~min}$ (Niki and Hiraga 1998). In contrast, at doubling times of 80 and 280 min, in cells with a single oriC focus, the focus localized mainly at mid-cell $(46.5 \%$ and $35.8 \%$ of oriC foci were localized at $40 \%-50 \%$ of the cell length, respectively: Fig. 1A, b,c). These results indicate that oriC is localized at midcell prior to initiation of chromosome replication in slowly growing cells.

\section{oriC segments migrate from mid-cell toward cell poles after replication}

As described above, a single oriC focus was localized mainly at the middle of cells in cultures growing at an 80 -min doubling time. This suggests that oriC is localized at mid-cell prior to initiation of replication, that is,
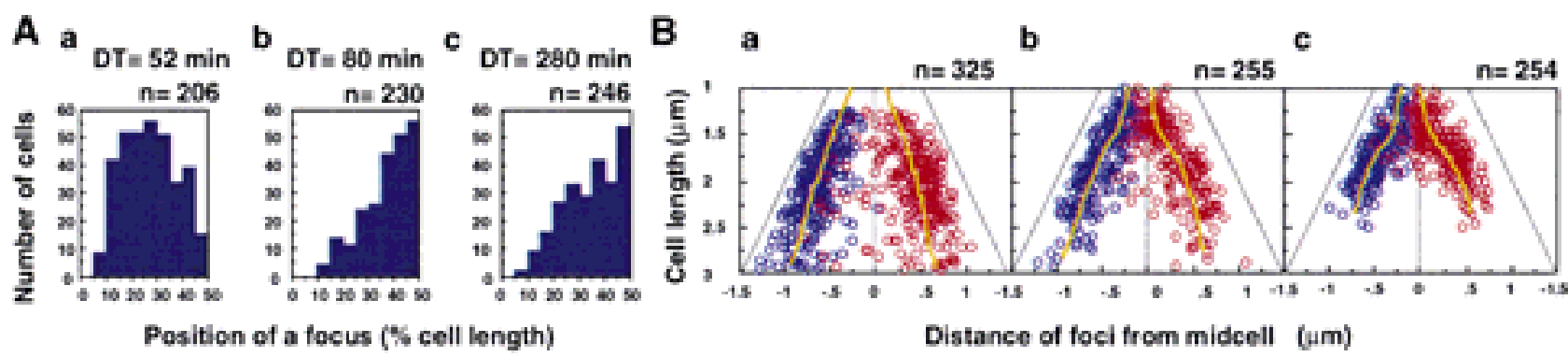

Figure 1. Localization of the oriC DNA segment in cells under various growth conditions. $(A)$ Statistical analysis of subcellular localization of a single fluorescent focus in cells. Cells were cultivated at $37^{\circ} \mathrm{C}$ in $\mathrm{M} 9$-glucose medium containing casamino acids with a doubling time of $52 \mathrm{~min}(a)$, M9-glucose containing proline with a doubling time of $80 \mathrm{~min}(b)$, and M9-sodium acetate containing proline with a doubling time of $280 \mathrm{~min}(c)$. The oriC region was detected by hybridization with the Cy-3 labeled oriC DNA probe. The histogram shows the distribution frequency of the focus in cells with a single focus $(a-c)$. Fraction of cells with a single oriC focus against cells with two foci was as follows: 1.1 (52 min), $2.0(80 \mathrm{~min})$, and $2.3(280 \mathrm{~min})$. (B) Statistical analysis of cells with two fluorescent foci of the oriC segment. Cells were cultivated at $37^{\circ} \mathrm{C}$ in $\mathrm{M} 9$-glucose medium containing casamino acids with a doubling time of $52 \mathrm{~min}(a)$, in M9-glucose and proline (doubling time: $80 \mathrm{~min}$ ) to mid-log phase $(b)$, and early stationary phase $(c)$. The positions of foci from mid-cell are plotted vs. cell length. In cells with two foci, the nearest oriC focus from a cell pole is shown as a blue circle; the other oriC focus is shown as a red circle. The yellow line indicates the regression line calculated by the Lowess method (tension, 50). The broken line indicates mid-cell; the solid line indicates the position of a pole. 
during the B period. We statistically analyzed the subcellular localization of oriC in cells with two oriC foci from the same exponentially growing culture. Two oriC foci were closely located in the vicinity of mid-cell in cells of newborn size, suggesting that oriC segments replicated at mid-cell (Fig. 1B, b,c). In contrast, two oriCs were located close to each cell pole in rapidly growing cells (Fig.1 B, a). Moreover, each focus was localized at a constant distance from the cell pole regardless of cell length, as described previously (Niki and Hiraga 1998). These results demonstrate that replicated oriC copies are abruptly separated from each other. On the other hand, in cases of slowly growing cells in both exponential and stationary phase, each oriC focus was localized at a constant distance from the cell pole only in longer cells $\mid \sim 1.5$ $\mu \mathrm{m}$ : Fig. 1B, b,c). The distribution of oriC foci in cells in both phases was bent at 1.4-1.6 $\mu \mathrm{m}$ of cell length. The results indicate that the replicated oriC copies were located near mid-cell until cells grew to $1.4-1.6 \mu \mathrm{m}$, and the oriC copies migrated abruptly in opposite directions toward each cell pole. The duplicated oriC copies and their flanking regions seem to be paired until an unidentified partitioning apparatus is activated.

\section{Subcellular localization of various chromosomal DNA} segments located at 5-min intervals on the chromosome map

To examine the localization of a whole chromosome in the cells, we used 22 DNA segments located at 5-min intervals on the genetic map of E. coli chromosome as fluorescent DNA probes with the FISH technique (Fig. 2). A distance of 5 min on the E. coli genetic map (0-100 $\mathrm{min}$ ) is equivalent to $230 \mathrm{~kb}$ of DNA. The DNA probes

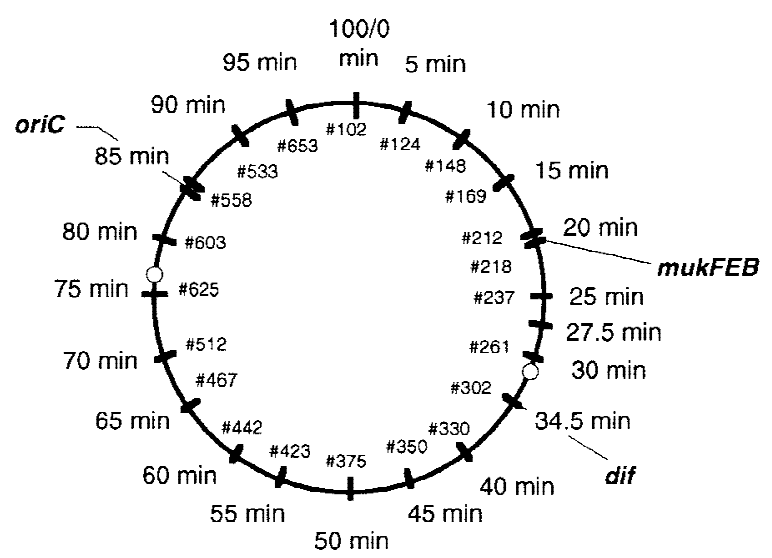

Figure 2. The map of E. coli chromosome with 23 DNA segments used as FISH probes. Positions of DNA probes derived from the Kohara phage library (Kohara et al. 1987) are indicated on the E. coli chromosome with map units in min (outside) and phage clone number (inside) according to EcoMap10 (Rudd 1998). Three DNA probes including oriC, dif, and the mukEFB operon are also shown. Open circles on the chromosome indicate the points of the inversion endpoints in strains KIN273and LN3214 (Cornet et al. 1996). were named according to their location on the genetic map; for example, the chromosome segment at $10 \mathrm{~min}$ on the chromosome was called the 10-min segment. In addition to these probes, we used a DNA segment (the 21-min segment) containing the mukEFB genes, whose products are involved in chromosome partitioning (Niki et al. 1991; Yamanaka et al. 1996).

We carried out a statistical analysis of the subcellular localization of fluorescent foci after classification of the cells in two groups: cells with a single focus and cells with two foci. The experimental results of the two groups are shown in Figures 3 and 4. In the case of cells with a single focus (Fig. 3), the oriC segment was predominantly localized in the middle region of cell, and the dif segment was also localized at mid-cell. Average cell length in cells with a single oriC focus was shorter than that in cells with two foci. On the other hand, cells with a single dif focus had a wide range of cell lengths. In long cells with a single dif focus, the focus was localized within a narrow region in mid-cell. These results are consistent with our previous observation that the terminal region is localized at mid-cell before cell division (Niki and Hiraga 1998). It should be noted that single foci of the oriC, 80-, 90-, 95-, and 100/0-min DNA segments were similarly localized in the middle region of cells with single foci (Fig. 3). In contrast, the 10-, 15-, and 20-min segments, which are located midway from oriC to dif on the genetic map (Fig. 2), were broadly distributed from a cell pole to midcell. The 60-, 65-, and 75-min segments were also distributed from a cell pole to midcell. The distribution patterns of these DNA segments change gradually, following the order of the genetic map.

In cells with two foci (Fig. 4), the two oriC copies were separated and localized near the cell poles, regardless of cell length. On the other hand, the two dif copies were localized close to each other at mid-cell, particularly in large cells. This indicates that both replicated dif copies are localized at mid-cell when the cell divides. For the other DNA segments, the distribution patterns changed gradually according to the order of the genetic map (Fig. 4), that is, the distance between the two foci decreased from oriC to dif, clockwise or counterclockwise.

\section{Chromosome segments showing similar localization patterns to oriC}

Interestingly, the 80-, 85-, 90-, 95-, and 100/0-min DNA segments were mainly localized at mid-cell, like the oriC segment (Fig. 3B). In particular, the localization of the 85 -min DNA segment, only $12 \mathrm{~kb}$ from oriC, was indistinguishable from that of the oriC segment. Moreover, in cells with two foci, these six DNA segments, including the oriC DNA segment, showed similar localization patterns, namely, localization at a nucleoid border near a pole (Fig. 4A). This suggests that when these segments are replicated, they are separated from each other and localized near the cell poles. Thus, the six DNA segments localized in this large 920-kb chromosome region show similar localization patterns; we call this the Ori domain. 


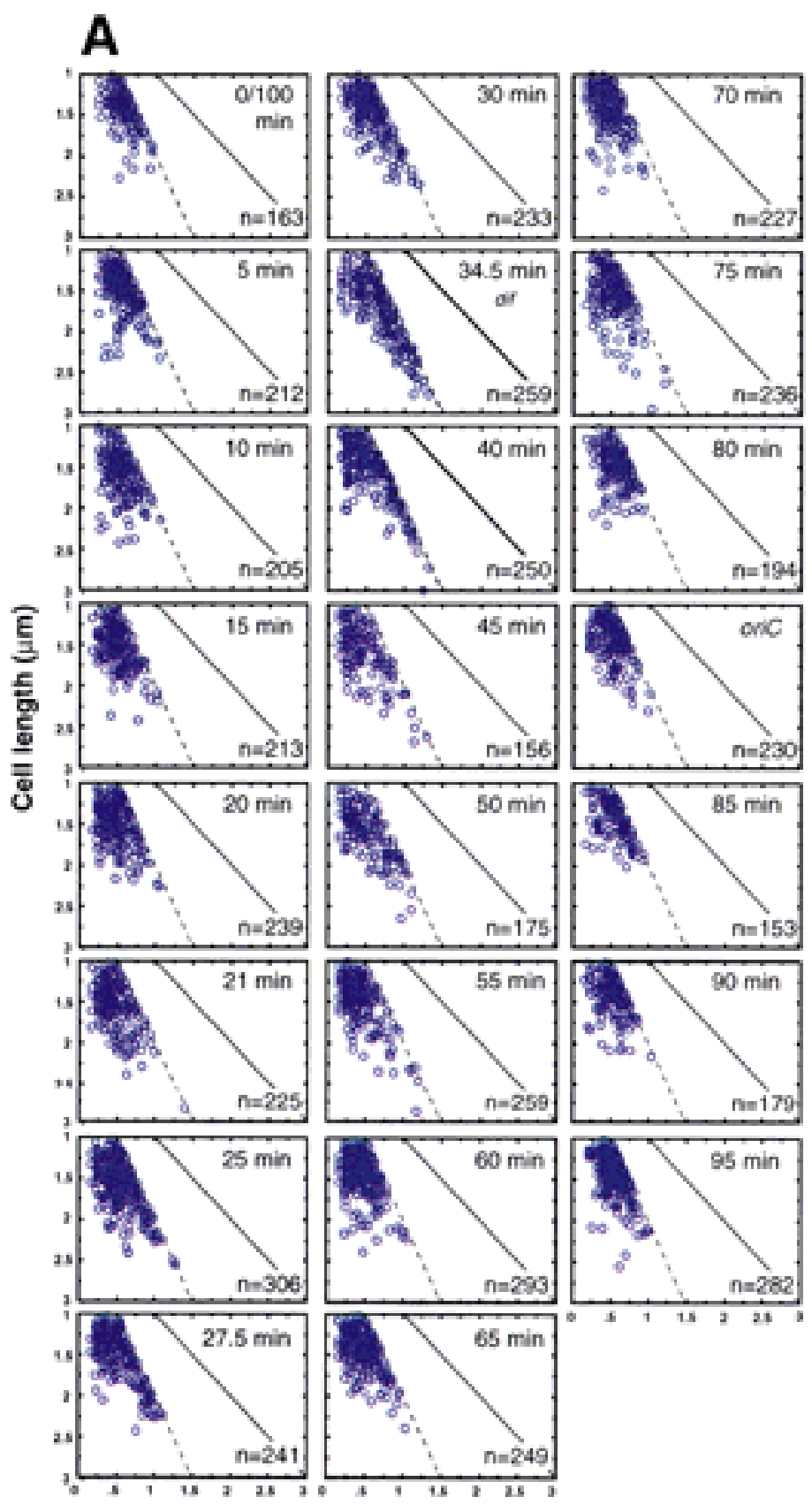

Distance of a focus from the nearest pole $(\mu \mathrm{m})$
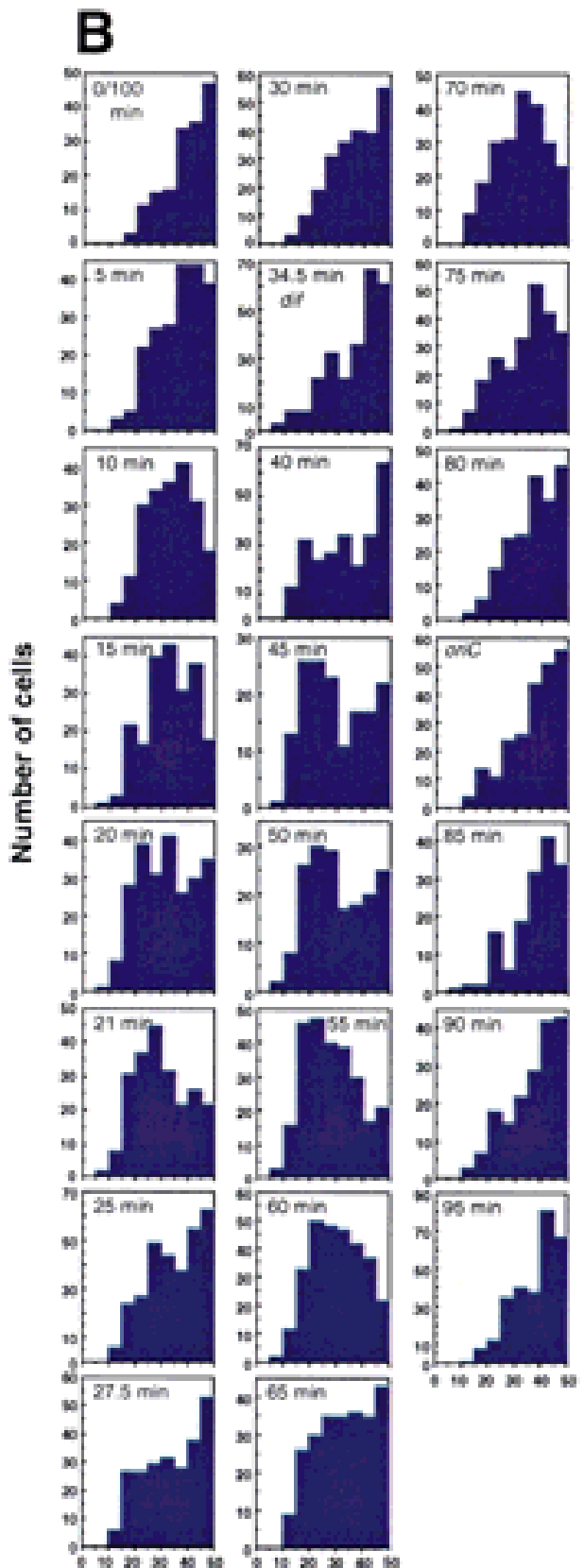

Position of a focus ( $\%$ cell length)

Figure 3. Localization of fluorescent foci of various chromosomal DNA segments in cells having a single focus in an exponentially growing culture. $(A)$ Cells were grown in M9-glucose containing proline (doubling time: $80 \mathrm{~min}$ ) to mid-log phase. Cells with a single fluorescent focus were analyzed statistically. The position of the fluorescent focus in cells with a single focus is plotted vs. cell length. The broken lines indicate the middle of the cell and the solid lines indicate the position of a pole. $(B)$ Histogram showing the distribution frequency of the foci in cells with a single focus.

Chromosome segments showing similar localization patterns to the dif segment

In this study we used the chromosomal segment including the dif site to detect the replication terminus region because the dif site is located at $180^{\circ}$ opposite oriC on the physical chromosome map. The dif site is a target of the XerCD site-specific recombinase for resolution of chromosome dimers, so the replicated dif sites are finally separated only during chromosome segregation (Louarn et al. 1994). The dif segment showed the same localization as the Ter DNA segment that we used previously to detect the replication terminus region (Niki and Hiraga 1998). 

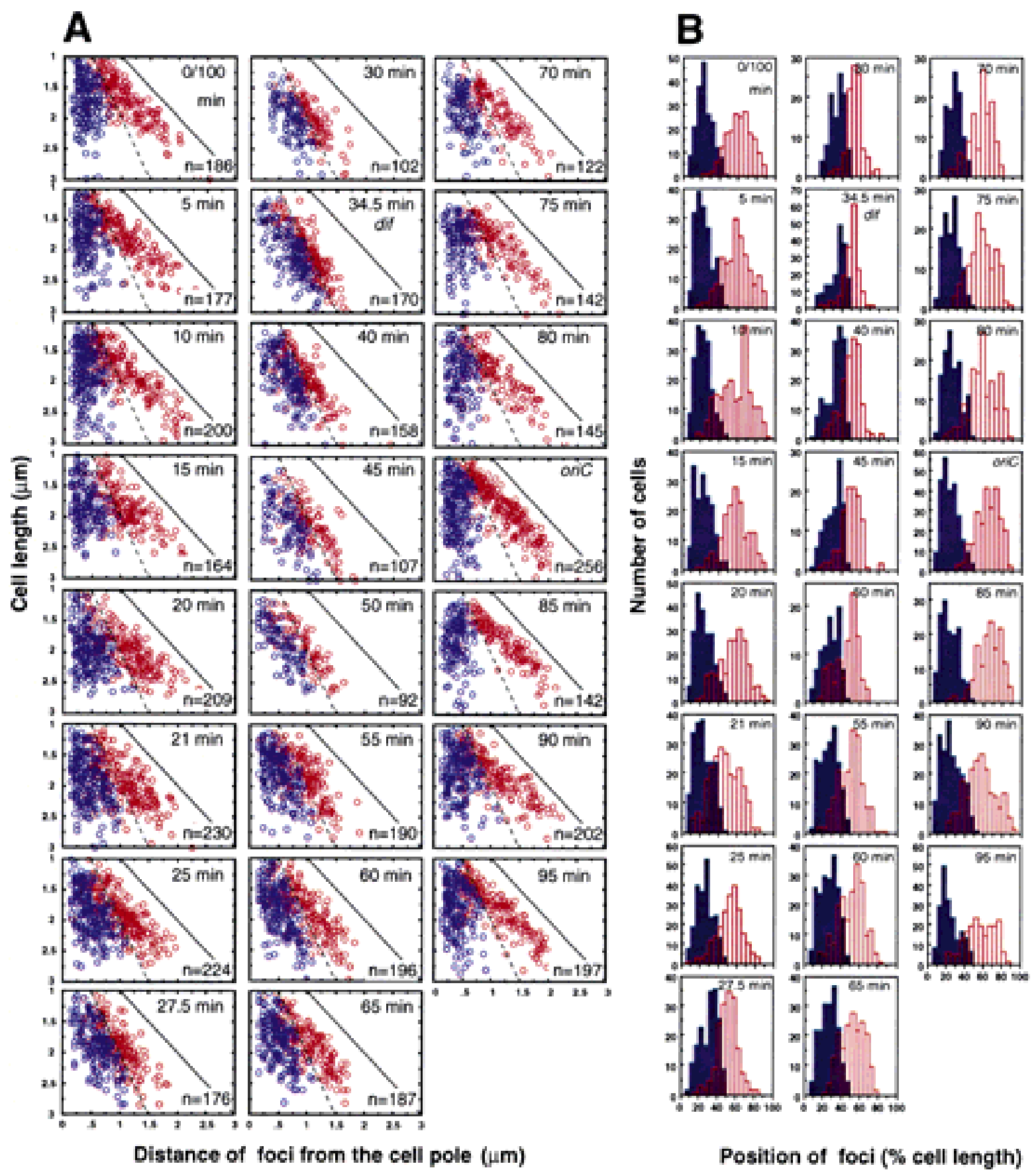

Position of foci ( $\%$ cell length)

Figure 4. Localization of fluorescent foci of various chromosomal DNA segments in cells with two foci in exponentially growing cultures. Cells with two fluorescent foci in the same culture as in Fig. 3 were statistically analyzed. $(A)$ Positions of both foci in cells with two fluorescent foci are plotted against cell length. The nearest oriC focus from a cell pole is shown as a blue circle; the other oriC focus from the same pole is shown as a red circle. $(B)$ Histogram showing the distribution frequency of both foci in cells with two foci.

In small cells with a single fluorescent focus of the dif segment (34.5-min), the foci were widely distributed between a cell pole and mid-cell (Fig. 3A). In contrast, a single focus of the dif segment in long cells was localized at or near mid-cell. On the other hand, in cells with two foci of the dif segment, the foci were mainly localized at or near mid-cell (Fig. 4). The 25-, 27.5-, 30-, 40-, and 45-min DNA segments were localized similarly to the dif segment. The localization pattern of these segments is characteristic of the $\sim 920-\mathrm{kb}$ replication terminus region, which we call the Ter domain.

\section{Localization of the replication origin and terminus DNA segments at mid-cell}

The results shown in Figures 1 and 3 indicate that the 
oriC DNA segment migrates from a cell pole to mid-cell during the B period, and the terminus segment also migrates toward mid-cell. Ultimately, the terminus segment colocalizes with oriC in the B period. To confirm the migration of these segments during the B period, we analyzed small cells for the positions of oriC and dif in a slowly growing culture (doubling time: $80 \mathrm{~min}$ ) by FISH simultaneously using oriC and dif DNA probes labeled with fluorescent compounds of different colors. Small cells, which might include newborn cells, were classified in three types as follows: (1) cells with a single oriC focus near one cell pole and a single dif focus near the other pole (Fig. 5A, a); (2) cells with a single oriC focus at mid-cell and a single dif focus near a cell pole (Fig. 5A, b), and (3) cells with the oriC and dif foci located at mid-cell (Fig. 5A, c). The statistical results revealed that ori $C$ foci were mainly localized at mid-cell and dif foci were localized from cell poles to mid-cell (Fig. 5B). We consider that these three types of cells show the process of migration of these segments during the B period, as ori $C$ is located near the old cell pole and dif near the new pole just after cells divide (Niki and Hiraga 1998).

\section{Localization of intermediate segments between oriC and dif}

The migration of chromosomal segments during the $\mathrm{B}$ periods was found not only for the ori $C$ and dif segments, but also for chromosomal segments between oriC and dif. The 20- and 70-min segments showed intermediate patterns of subcellular localization between oriC and dif, as shown in Figures 3 and 4. When localization of the 20and 70-min segments was analyzed simultaneously, their foci were separated from each other along the long axis of cell length (Fig. 5A, d,e). In a small fraction of cells, both foci were localized near mid-cell (Fig. 5A, f). However, statistical results revealed that foci of the 20and 70-min segments were mainly separated from each other in short-length cells (Fig. 5C). After duplication of the 20- and 70-min segments, each focus was localized between the cell pole and mid-cell in long-length cells in which oriC copies may be localized at each cell pole and dif segment(s) at mid-cell (Fig. 5A, g). Localization of the 20 -min segment relative to the oriC or dif segment showed that the 20-min segment tended to separate from oriC and dif (Fig. 5A, h,i, D,E).

\section{Subcellular localization of the DNA segments in stationary-phase cells}

In exponentially growing cells, DNA replication and RNA transcription occur actively throughout the cell cycle; thus, the nucleoid is in an open, partially relaxed state. In contrast, in stationary phase, the nucleoid becomes more compact because DNA replication and RNA transcription slow down. We analyzed statistically cells of early stationary phase (at $1.5 \mathrm{hr}$ after entry into stationary phase) for the position of a single focus or two foci using the same set of DNA probes for FISH, as

\section{A}
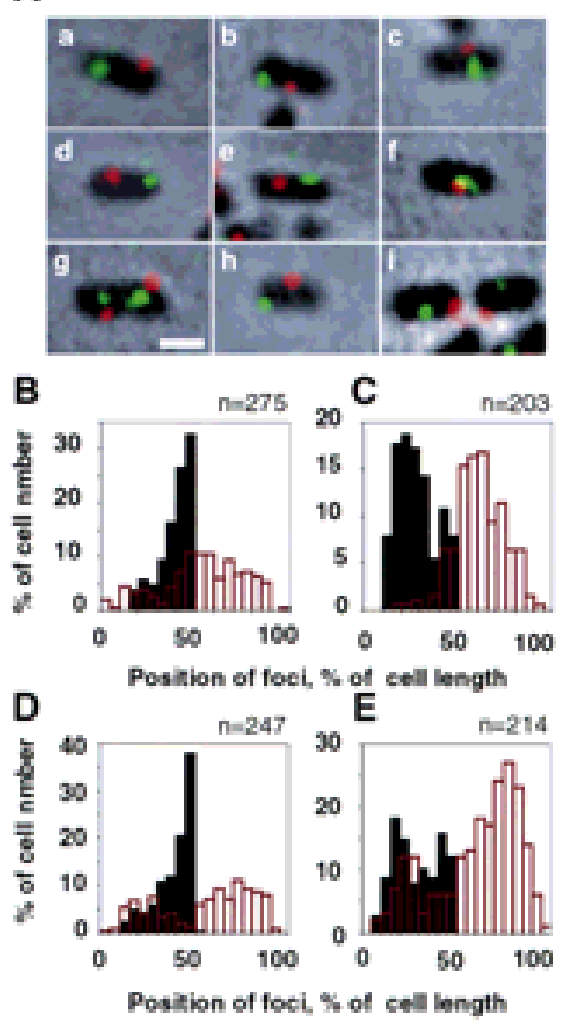

Figure 5. Localization of two segments in the same cells. $(A)$ Cells were grown as described in the legend to Fig. 3 and simultaneously hybridized with the fluorescein-labeled DNA probes (green) and the Cy3-labeled DNA probes (red). $(a-c)$ Cy3-oriC and fluorescein-dif; $(d-g)$ Cy3-20- and fluorescein-70-min segments; (h) Cy3-oriC and fluorescein-20-min segment; (i) Cy3dif and fluorescein-20-min segment. All photos combine the phase-contrast micrograph with the fluorescent micrograph for FISH in the same cell. We measured distance between the center of the Cy3 focus and the nearest pole and the distance between that of the fluorescein focus from the same pole. $(B-E)$ Cells were analyzed statistically. The histogram shows the distribution frequency of both foci in cells with two foci. (B) Localization of oriC (black) and dif (red); $(C)$ localization of the 20 (black) and 70-min segments (red); (D) localization of oriC (black) and the 20-segment (red); (E) localization of dif (black) and the 20-min segment (red). Scale bar, $1 \mu \mathrm{m}$.

shown in Figure 2. There were no significant differences in the subcellular localization of chromosome segments between exponential phase and early stationary phase (data not shown), except for the oriC segment, as shown in Figure 1B, b. In cells with a single focus, DNA segments between 70 and 100/0 min were mainly localized at mid-cell. This same chromosomal region was also localized at mid-cell in stationary-phase cells.

Independent localization of the dif segment in the large inversion

Bipolar migration of replicated oriC copies suggests that 
there is a cis-acting region(s) for positioning in the Ori domain. During the B period, the Ter domain migrates from the new pole, created by cell division, to mid-cell. Does the migration of the Ter domain occur became of an active mechanism or is it merely due to reorganization of the chromosomal structure after migration of the Ori domain?

To address this question, we analyzed the mutant strain KIN273, which has a chromosome inversion placing the replication terminus region near oriC on the chromosome. This large inversion was created by recombination between two copies of the insertion sequence IS5, IS5F at $29 \mathrm{~min}$, and IS5T at $78 \mathrm{~min}$ on the chromosome, resulting in the transposition of the dif site to a new position $11 \mathrm{~min}(\sim 500 \mathrm{~kb})$ counterclockwise of oriC (see Fig. 2; Louarn et al. 1985). We examined the subcellular localization of the oriC segment in the wild-type strain CB0129 and the large inversion mutant KIN273. In the mutant cells, a single fluorescent focus of oriC was located at a cell pole (Fig. 6B, a) or two foci were localized near the poles (Fig. 6B, b-d), as in the wild-type strain (data not shown). When oriC segments were localized normally (at cell poles) in the inversion mutant cells, replicated dif segments were localized at mid-cell (Fig. 6B, b-d), indicating that the dif segments are capable of localizing at their normal positions. Taken together, these results on oriC and dif in the inversion mutant suggest that the Ori domain and the Ter domain have their own positioning systems.

\section{Formation of anucleate cells in the large inversion mutant by mis-segregation}

We examined chromosome segregation in the inversion mutants by DAPI staining (Fig. 6A). Filamentous cells, which were caused by chromosome dimerization, were observed rarely, indicating that dif and XerCD recombination system functioned normally, as described by Cornet et al. (1996). However, the inversion strain produced anucleate cells at high frequency (Table 1). Anucleate cells of normal cell length were produced at cell division (Fig. 6A, b). In some cells with a deep constriction, one daughter cell had two nucleoids and the other none (Fig. 6A, c-e). Faint fluorescence of chromosomal DNA stained with DAPI remained on one side of the deep constriction. This phenomenon, the so-called Guillotine effect, is often observed in chromosome partitioning mutants mukF, mukE, and mukB (Niki et al. 1991; Yamanaka et al. 1996). Our present microscopic observations demonstrate that the chromosome rearrangement in the inversion mutants causes mis-segregation of daughter chromosomes into daughter cells.

The inversion mutant grew more slowly than wildtype cells; doubling time of the mutant was $60 \mathrm{~min}$ in $\mathrm{L}$-medium at $37^{\circ} \mathrm{C}$ and that of wild type was $28 \mathrm{~min}$. However, the length of the $\mathrm{C}$ period of the mutant is not known yet. No difference concerning production of anucleate cells was found for tus ${ }^{+}$and the tus null mutation (data not shown). The Tus protein binds to Ter sites and arrests replication folks (Hill 1996). Therefore,
A

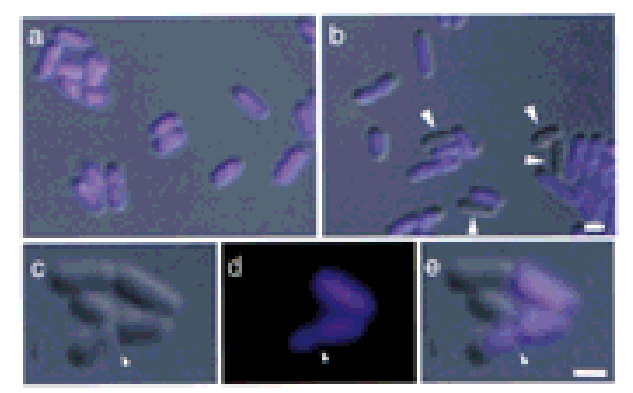

B

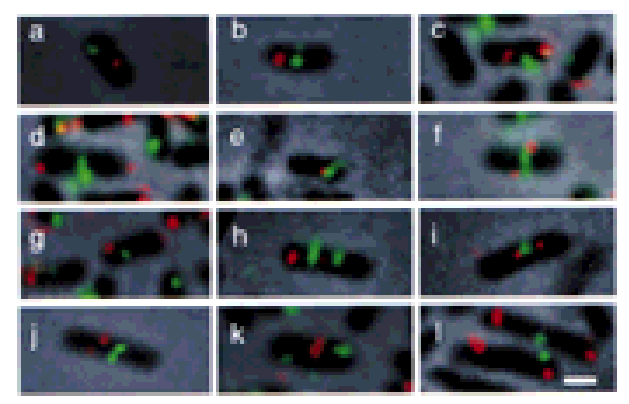

C

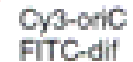

Merge

DAPI
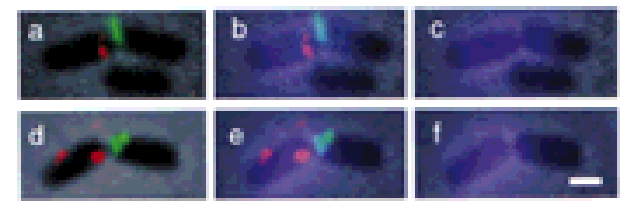

Figure 6. Localization of the oriC and dif segments on segregating chromosomal DNA in the large inversion cells. Cells were grown to mid-log phase at $30^{\circ} \mathrm{C}$ in $\mathrm{M} 9$-glucose minimal medium. (A) Visualization of segregating chromosomal DNA in large inversion cells. Fixed cells were stained with DAPI to detect chromosomal DNA. (a) Wild-type CB0129 cells, $(b-e)$ KIN273 cells. Cells are shown in Nomarski DIC images combined with fluorescent DAPI images $(a, b, e),(c)$ DIC images, $(d)$ fluorescent DAPI images. Arrowheads indicate anucleate cells $(b)$ or the Guillotine effect $(c-e) .(B, C)$ All images were combined of the fluorescent and the phase-contrast micrograph. $(B)$ Localization of the oriC and dif segments in KIN273 is simultaneously shown in images of the fluorescent micrograph for the Cy3-labeled oriC probe and the fluorescein-labeled dif probe. $(C)$ Localization of the oriC and dif segments in KIN273 is simultaneously shown in images of the fluorescent micrograph for the Cy3-labeled oriC probe, the fluorescein-labeled dif probe, and fluorescent DAPI micrograph. Scale bars, $1 \mu \mathrm{m}$.

it is likely that the blocking of replication folk does not affect anucleate cell production in the inversion mutants.

\section{Mispositioning of oriC and dif DNA segments in the large inversion mutant}

oriC and dif segments were frequently localized abnormally in the mutant cells, as follows. oriC and dif foci 
Table 1. Frequency of anucleate cells and mis-positioning in the inversion mutants

\begin{tabular}{llcl}
\hline Strain $^{\mathrm{a}}$ & \multicolumn{1}{c}{ Genotype } & $\begin{array}{c}\text { Anucleate } \\
\text { cells }\end{array}$ & \multicolumn{1}{c}{ Mis-positioning } \\
\hline CB0129 & Inv $^{+}$ & $0 \%(0 / 1407)$ & $10.6 \%(9 / 83)$ \\
KIN273 & Inv (29-78) & $13.9 \%(227 / 1631)$ & $34.6 \%(36 / 101)$ \\
\hline
\end{tabular}

${ }^{\mathrm{a} C e l l s}$ were grown in $\mathrm{M} 9$ minimal medium at $30^{\circ} \mathrm{C}$.

${ }^{b}$ In cells with two oriC foci, both foci were localized at half of a cell.

were colocalized at a cell pole (Fig. 6B, e). Two oriC foci were localized near mid-cell where dif foci localized (Fig. $6 \mathrm{~B}, \mathrm{f}, \mathrm{i}-\mathrm{k})$. One oriC focus was localized between dif foci near mid-cell; the other at or near a cell pole (Fig. 6B, h). Chromosomal DNA and two oriC foci remained on one side of the constriction (Fig. 6C). Thus, the proper positioning of ori $C$ copies was often disturbed in the mutant strain (Fig. 7B; Table 1). In addition, the dif segment was frequently mispositioned in the mutant (Fig. 7D). The dif segments tended to be localized at mid-cell; however, the localization was broader than that in wild-type cells (Fig. 7C). A dif focus was localized near a cell pole but not at mid-cell (Fig. 6B, g,1). As shown in Figure 6B, k, two oriC foci were localized at mid-cell and each dif focus near cell poles, just the opposite of the normal positions. In some cells, a large part of the chromosomal DNA was located on one side of a deep constriction and the two dif segments were localized at the constriction

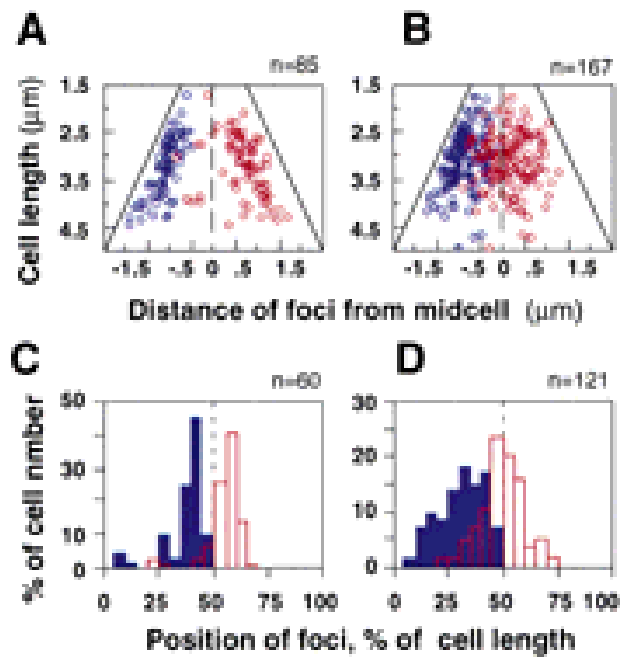

Figure 7. Localization of the oriC and dif segments in the inversion mutant. Statistical analysis of cells with two fluorescent foci of the oriC $(A, B)$ or dif segment $(C, D)$. Wild-type cells $(A, C)$ and the inversion mutant, $\operatorname{KIN} 273(B, D)$ were cultivated at $30^{\circ} \mathrm{C}$ in $\mathrm{M} 9$-glucose medium. $(A, B)$ The positions of foci from mid-cell are plotted vs. cell length. In cells with two foci, the nearest oriC focus from a cell pole is shown as a blue circle; the other oriC focus is shown as a red circle. $(C, D)$ Histogram showing the distribution frequency of both foci in cells with two foci. The broken line indicates mid-cell; the solid line indicates the position of a pole. site (Fig. 6C, a-c) or on the chromosome-less side near the constriction (Fig. 6C, d-f). This result suggests that a small DNA segment including dif had been cut off from the chromosome by septum closure. In the inversion mutant, the oriC and dif segments could be localized at their normal positions. In many cases $(30 \%)$, however, it was likely that positioning of the oriC and dif segments interfered with each other.

\section{Discussion}

The Ori and Ter domains in chromosome organization and positioning

We have demonstrated that a large chromosome region including oriC-an $~ 900$-kb DNA segment $(20 \%$ of the chromosome; Fig. 8A)-exhibits bipolar migration toward each cell pole during ongoing replication. On the other hand, a large chromosomal region including dif, also $\sim 900 \mathrm{~kb}$ in length, is localized near mid-cell during replication and also during the $\mathrm{D}$ period, until the cell divides. Other regions are localized between the Ori and Ter domains in the cell according to the order of the $E$. coli chromosome map. Upon separation of daughter cells, the Ori domain is localized near an old pole and the Ter domain near a new pole at the division site. However, before initiation of chromosome replication, the Ori domain moves to mid-cell during the B period. Following the migration of the Ori domain, the Ter domain also can migrate toward mid-cell. Ultimately, it appears that whole chromosomes rotate during the B period in some cells (Fig. 8B).

A natural environment is not always suitable for rapid growth of cells in E coli. Although the E. coli cells in the mucus layer grow with a doubling time of 30-80 min, they are static in the lumen (Poulsen et al. 1995). Because $E$. coli cells grow very slowly in the anaerobic environment of the large intestine, the B period is a major phase in the $E$. coli life cycle in its natural environment.

\section{Ring structure in the E. coli chromosome}

Our results on ordered chromosome localization and chromosome rotation strongly suggest that the E. coli chromosome is organized in a compact ring structure aligned with the E. coli map. Interestingly, such a compact ring structure could be observed by fluorescence microscopy in DAPI-stained E. coli cells (Fig. 8C). A small percentage of cells grown exponentially at $22^{\circ} \mathrm{C}$ had a ring-like chromosome (Fig. 8C, a,b). When cells were grown at $37^{\circ} \mathrm{C}$ and in early stationary phase at $22^{\circ} \mathrm{C}$, such a ring structure was hardly ever observed. In addition, more clearly circular chromosomes were observed in mukB-disrupted mutant cells at $22^{\circ} \mathrm{C}$ (Fig. $8 \mathrm{C}$, c, d). Because MukB may be a member of the SMC family and involved in the pathway of chromosome folding in $E$. coli, it is likely that compaction of folded chromosome is relaxed in the mutants. In other microorganisms, a ring structure of chromosomes was reported under special 

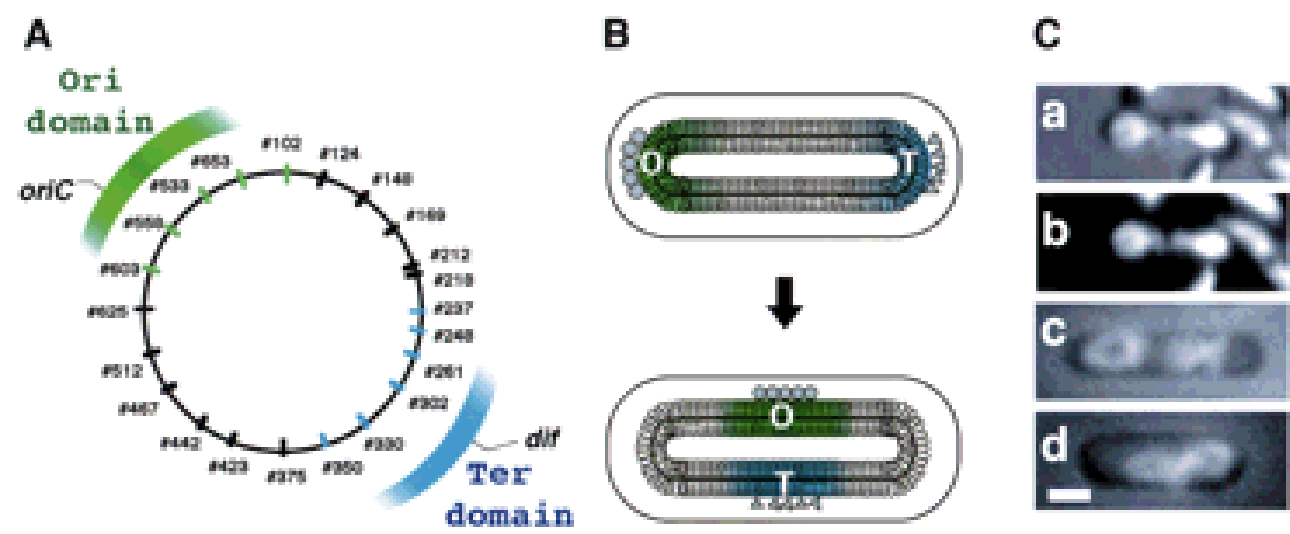

Figure 8. Domains, organization, and dynamics of the bacterial chromosome. (A) Organization of the Ori and Ter domains. The DNA segments that showed similar localization patterns to that of the oriC segment are shown in green. The DNA segments that showed similar localization patterns to that of the dif segment are shown in blue. (B) A model of bacterial chromosome structure in vivo. Long circular bacterial chromosomal DNA may be folded by an unknown mechanism to form a compact ring structure, in which the DNA segments are arranged according to their chromosome map positions. The Ori (green, O) and Ter domains (blue, T) are localized near or at the cell poles in newborn cells $(t o p)$. In the $B$ period, both the domains migrate and are localized at mid-cell (bottom). Factors (gray circles) may bind specifically to cis-acting sites of the Ori domain; other factors (gray triangles) may bind to another type of cis-acting site of the Ter domain. The two groups of factors may participate in cell cycle-dependent positioning of the Ori and Ter domains. $(C)$ Fluorescent microscopy of DAPI-stained cells. Wild-type $(a, b)$ and mukB-disrupted mutant $(c, d)$ that were grown at $22^{\circ} \mathrm{C}$ were stained with DAPI according to Hiraga et al. (1989). (a,c,d) Images of fluorescence-phase contrast-combined microscopy. (b) Image of fluorescence microscopy in the same field as $a$. Scale bars, $1 \mu \mathrm{m}$.

conditions. The B. subtilis chromosome in developing spores has a doughnut-like ring structure (Pogliano et al. 1995). Circular chromosomes have also been observed in M-phase-arrested fission yeast cells that have lost all telomeric DNA sequences by mutations affecting telomere maintenance (Naito et al. 1998). We cannot completely eliminate the possibility that the ring structure of $E$. coli chromosomes in fixed cells is an artifact or abnormal state. Nevertheless, this observation is consistent with the ordered chromosome localization and chromosome rotation that were demonstrated by the FISH method.

\section{Formation of the domains and bipolar} chromosome migration

Bipolar migration of oriC segments in E. coli and B. subtilis is established during chromosome segregation. Formation of two Ori domains on a replicating chromosome may play an important role in the bipolar migration. During spore formation in B. subtilis, a $0.93-$ to $1.4-\mathrm{Mb}$ chromosome region including oriC is localized at a cell pole in the prespore compartment $\mathrm{Wu}$ and Errington 1998). Such regular positioning of the oriC region at the cell pole is prevented at the onset of sporulation in a spoOI mutant (Sharpe and Errington 1996). Interestingly, eight binding sites for the SpoOJ protein are found in the chromosome region $(20 \%)$ including oriC (Lin and Grossman 1998). Spo0J-Gfp is observed as discrete fluorescent foci, which show bipolar migration in sporulation and vegetative growth (Glaser et al. 1997; Lin et al. 1997). The B. subtilis Spo0J protein is a homolog of SopB/ParB, products of plasmid-encoded partition genes, from E. coli plasmids such as $\mathrm{F}$ and $\mathrm{P} 1$.

On the other hand, the genome of E. coli has neither
SopA/ParA nor SopB/ParB homologs. We speculate that uncharacterized $E$. coli factors are responsible for the subcellular positioning of the Ori domain. The Ori domain presumably has cis-acting DNA sequences that function as centromere when unidentified partition proteins bind to them. The Ter domain may have another positioning system, also controlled by specific proteins and cis-acting DNA sequences. The chromosome segments of the Ter domain in a tus-disrupted mutant localized as in a wild-type strain (H. Niki et al., unpubl.). Thus, the absence of the Tus protein does not affect the positioning of the Ter domain.

During bipolar migration of oriC, the two copies of replicated ori $C$ migrate in opposite directions, from midcell to each cell pole, in slowly growing cells (Fig. 1B, $\mathrm{b}, \mathrm{c})$. In contrast, one copy of replicated oriC migrates from one cell pole to the other in rapidly growing cells (Fig. 1B, a; Niki and Hiraga 1998). The former pattern of movement is observed frequently in B. subtilis cells grown under steady-state conditions (Sharpe and Errington 1998; Webb et al. 1998). In B. subtilis, oriC is replicated at or near mid-cell and the two replicated copies migrate toward the cell poles until they are separated from each other by a fixed distance (Sharpe and Errington 1998). In E. coli, under both slow and rapid growing conditions, an active mechanism for bipolar migration may separate the two replicated oriC copies at a constant distance. Thus the "ruler-like" behavior of the active mechanism (Sharpe and Errington 1999) is common to the segregation of specific chromosome sites and plasmids.

Role of the Ter domain

In the chromosome-partitioning process, the replicated 
Ori domains are first positioned near each cell pole. This step is very critical in delivering replicated chromosomes to each daughter cell. The Ter domain, on the other hand, is positioned at mid-cell and contributes directly to the resolution of a chromosome dimer at the dif site. An odd number of recombinations between daughter chromosomes during replication results in the formation of a chromosome dimer molecule, and the dimer prevents chromosome segregation into the two daughter cells. To ensure resolution of chromosome dimers, a site-directed resolution system, dif/XerCD, works at the final step of physical separation of daughter chromosomes. The dif site is functional only in a restricted region of the Ter domain (Cornet et al. 1996; Kuempel et al. 1996). It is thought that some special organization of the chromosome, including the dif site, may be essential for normal chromosome dimer resolution at dif (Louarn et al. 1994). Moreover, FtsK is localized at the septum and involved in the dif/XerCD resolution system (Steiner et al. 1999). Presumably, organization of the Ter domain helps the dif/XerCD resolution system to function at the restricted chromosome site, and positioning of the domain at mid-cell makes the dif site accessible to FtsK.

\section{Chromosome organization in the inversion mutants}

Bacterial chromosomal DNA is folded to form a compact structure with 40-50 negatively supercoiled chromosomal domains. Such supercoiling is restrained into 40to $100-\mathrm{kb}$ DNA segments by connecting the bases of the supercoiled loops (Pettijohn 1996). Although supercoiling of DNA loops by DNA gyrase is important for compaction, the folding mechanism is not clearly understood. A compacted chromosome may retain torsional tension in its structure. We speculate that the torsional tension of organized chromosomes is a major reason for mis-segregation in the mutants with the inversion of the large chromosomal region. The distance between oriC and dif in the inversion mutants is shorter than in the wild-type strain. The condensed spacer region between oriC and dif may be insufficient for localization at their proper positions, a cell pole and mid-cell, on cell division. Nevertheless, the spacer region can be expanded to allow bipolar positioning of duplicated Ori domains near each cell pole and Ter domains at mid-cell (Fig. 6B, b-d). However, if an expanded spacer region is shrunk by torsional tension, the Ori domain near each cell pole is pulled and localized forcibly near the Ter domain (Fig. $6 \mathrm{~B}, \mathrm{f}, \mathrm{i}, \mathrm{j}$ ) and similarly for the Ter domain (Fig. 6B, g, 1). In the former case, an arrangement with one of the Ori domains is localized near a cell pole and the other at mid-cell or both in half of a cell will cause mis-segregation of daughter chromosomes (Fig.6C).

It is possible that production of anucleate cells in the large inversion mutant could be due to secondary effects caused by alternations in either replication or transcription, or both. However, the fact that ori and dif are frequently mispositioned in the mutant suggests that cor- rect positioning of the Ori and Ter domains plays an important role in segregation process.

\section{Materials and methods}

Bacteria and culture conditions

All E. coli strains used in this study were K-12 derivatives, as follows: CSH50 [ara, $\Delta$ (lac-pro), strA, thi (Miller 1972)]; CB0129 $\left[\mathrm{F}^{-} \mathrm{W} 1458 \mathrm{deoB}\right.$ or $C$ leu thi supD], LN3214 [the same as CB0129 except Inv(29-78), dnaA46, tus::bla]; and KIN273 [the same as CB0129 except $\operatorname{Inv}(29-78)$, tnaA::Tn10, tus::bla]. KIN273 was constructed from LN3214 by P1 cotransduction of $d n a A^{+}$with $\operatorname{tn} a A:: \operatorname{Tn} 10$ for this study. Inv(29-78) in KIN3214 was confirmed using PCR on inverted IS5 sequences, IS5F at 29 min and IS5T at $78 \mathrm{~min}$. CSH50 cells were cultivated at $37^{\circ} \mathrm{C}$ in M9 medium with glucose $(0.5 \%)$ and thiamine $(2 \mu \mathrm{g} / \mathrm{ml})$. CB0129 and derivatives were cultivated at $30^{\circ} \mathrm{C}$ in $\mathrm{M} 9$ medium. We added proline $(50 \mu \mathrm{g} / \mathrm{ml})$ for CSH50 and leucine $(50 \mu \mathrm{g} / \mathrm{ml})$ and thymine $(25 \mu \mathrm{g} / \mathrm{ml})$ for CB0129 and derivatives.

\section{DAPI staining}

To fix cells, an equal volume of fixation solution [methanol: acetic acid (3:1)] was added directly to a bacterial culture growing exponentially in $\mathrm{M} 9$ medium at $37^{\circ} \mathrm{C}$. The fixed cells were collected by centrifugation and resuspended in $1 / 10$ volume of fixation solution. The fixed cells were dropped onto a poly-Llysine-coated slide. To observe nucleoid shape, cells were treated according to the procedure described previously (Hiraga et al. 1989). The dried cells were mounted with the medium $190 \%$ glycerol, $1 \mathrm{mg} / \mathrm{ml}$ p-phenylenediamine dihydrochloride, $0.15 \mu \mathrm{g} / \mathrm{ml}$ DAPI). Nomarski differential interference contrast (DIC) images were taken by a Nikon E-800 microscope with Plan Fluor DIC.

\section{Fluorescent probes for in situ hybridization}

To detect the replication origin DNA segment of the E. coli chromosome in fixed cells by in situ hybridization, we used the oriC DNA segment containing mioC, oriC, gidAB, and part of atpI, which was amplified from CSH50 chromosomal DNA by PCR (Niki and Hiraga 1998). We used Kohara's $\lambda$ phage clone 302 carrying dif as the dif DNA segment (Kohara et al. 1987) to detect the replication terminus region. Other Kohara $\lambda$ phage clones used in this study are shown in Figure 2. The cloned chromosomal DNA segments of these $\lambda$ clones were amplified by PCR and labeled with Cy3-dCTP or fluorescein-11-dUTP according to the procedure described previously (Niki and Hiraga et al. 1998).

\section{FISH and image analysis}

Fluorescence in situ hybridization (FISH) was carried out according to the procedure described previously (Niki and Hiraga 1997). All images were recorded with a cooled CCD camera, C5810-01 (Hamamatsu Photonics K.K., Hamamatsu, Japan), using a phase-contrast and fluorescence microscopy system (Nikon). The images were transferred directly to a Power Macintosh and processed using Adobe Photoshop 4.0-J software. To measure cell length and distance of fluorescent focus from a cell pole on a monitor, we used an image analyzing software, MacScope, version 2.5.5 (Mitani Corp., Japan). Focus position is the 
distance between the center of the focus and the nearest cell pole. In a histogram, focus position is calculated as the ratio (\%) of the distance between the nearest pole and the focus against cell length and expressed as a percentage. Statistical analysis was carried out using StatView 5.0-J software (SAS Institute Inc., Cary, NC, USA)

\section{Acknowledgments}

We are grateful to K. Hayashi, T, Horiuchi, and H. Mori for providing the Kohara phage DNAs, T. Onogi for photomicrographs of DAPI-stained cells, and J.-M. Louarn for providing the inversion mutants. We thank Richard D'Ari for critical reading of the manuscript and comments. This work was supported by a grant from the Grants-in-Aid for Scientific Research (C), Grants-in-Aid for Scientific Research on Priority Areas (A) from the Ministry of Education, Science, Sports, and Culture of Japan, and a grant from the Human Frontiers Science Program (RG386/95M). H. N. is supported by Ciba-Geigy Foundation (Japan) for the Promotion of Science.

The publication costs of this article were defrayed in part by payment of page charges. This article must therefore be hereby marked "advertisement" in accordance with 18 USC section 1734 solely to indicate this fact.

\section{References}

Britton, R.A., D.C. Lin, and A.D. Grossman. 1998. Characterization of a prokaryotic SMC protein involved in chromosome partitioning. Genes \& Dev. 12: 1254-1259.

Cornet, F., J. Louarn, J. Patte, and J.M. Louarn, 1996. Restriction of the activity of the recombination site dif to a small zone of the Escherichia coli chromosome. Genes \& Dev. 10: $1152-1161$.

Glaser, P., M.E. Sharpe, B. Raether, M. Perego, K. Ohlsen, and J. Errington. 1997. Dynamic, mitotic-like behavior of a bacterial protein required for accurate chromosome partitioning. Genes \& Dev. 9: 1160-1168.

Gordon, G.S., D. Sitnikov, C.D. Webb, A. Teleman, A. Straight, R. Losick, A.W. Murray, and A. Wright. 1997. Chromosome and low copy plasmid segregation in E. coli: Visual evidence for distinct mechanisms. Cell 90: 1113-1121.

Hill, T.M. 1996. Features of the chromosomal terminus region. In Escherichia coli and Salmonella typhimurium: Cellular and molecular biology (ed. F.C. Neidhardt, et al.), pp. 1602 1614. American Society for Microbiology, Washington, D.C.

Hiraga, S., H. Niki, T. Ogura, C. Ichinose, H. Mori, B. Ezaki, and A., Jaffé. 1989. Chromosome partitioning in Escherichia coli: Novel mutants producing anucleate cells. J. Bacteriol. 171: 1496-1505.

Hirano, T. 1999. SMC-mediated chromosome mechanics: a conserved scheme from bacteria to vertebrates? Genes \& Dev. 13: 11-19.

Kaidow, A., M. Wachi, J. Nakamura, J. Magae, and K. Nagai. 1995. Anucleate cell production by Escherichia coli $\Delta$ hns mutant lacking a histone-like protein, H-NS. J. Bacteriol. 177: 3589-3592.

Kellenberger, E. 1990. Intracellular orgamization of the bacterial genome. In The bacterial chromosome (ed. J. Drlica and M. Riley), pp. 173-186. American Society for Microbiology, Washington, D.C.

Kohara, Y., K. Akiyama, and K. Isono. 1987. The physical map of the whole E. coli chromosome: Application of a new strat- egy for rapid analysis and sorting of a large genomic library. Cell 50: 495-508.

Kuempel, P., A. Hogaard, M. Nielsen, O. Nagappan, and M. Tecklenburg. 1996. Use of a transposon (Tndif) to obtain suppressing and nonsuppressing insertions of the dif resolvase site of Escherichia coli. Genes \& Dev. 10: $1162-1171$.

Lemon, K.P. and A.D. Grossman. 1998. Localization of bacterial DNA polymerase: evidence for a factory model of replication. Science 282: 1516-1519.

Lin, D.C., and A.D. Grossman. 1998. Identification and characterization of a bacterial chromosome partitioning site. Cell 92: 675-685

Lin, D.C.-H., P.A. Levin, and A.D. Grossman. 1997. Bipolar localization of a chromosome partition protein in Bacillus subtilis. Proc. Natl. Acad. Sci. 94: 4721-4726.

Louarn, J., F. Cornet, V. Francois, J. Patte, and J.M. Louarn. 1994. Hyperrecombination in the terminus region of the Escherichia coli chromosome: Possible relation to nucleoid organization. J. Bacteriol. 176: 7524-7531.

Louarn, J.M., J.P. Bouche, F. Legendre, J. Louarn, and J. Patte. 1985. Characterization and properties of very large inversions of the $E$. coli chromosome along the origin-to-terminus axis. Mol. \& Gen. Genet. 201: 467-476.

Melby, T.E., C.N. Ciampaglio, G. Briscoe, and H.P. Erickson. 1998. The symmetrical structure of structural maintenance of chromosomes (SMC) and MukB proteins: Long, antiparallel coiled coils, folded at a flexible hinge. J. Cell. Biol. 142: $1595-1604$.

Miller, J.H. 1972. Experiments in molecular genetics. Cold Spring Harbor Laboratory, Cold Spring Harbor, NY.

Moriya, S., E. Tsujikawa, A.K. Hassan, K. Asai, T. Kodama, and N. Ogasawara. 1998. A Bacillus subtilis gene-encoding protein homologous to eukaryotic SMC motor protein is necessary for chromosome partition. Mol. Microbiol. 29: 179187.

Naito, T., A. Matsuura, and F. Ishikawa. 1998. Circular chromosome formation in a fission yeast mutant defective in two ATM homologues. Nat. Genet. 20: 203-206.

Niki, H., and S. Hiraga. 1997. Subcellular distribution of actively partitioning F plasmid during the cell division cycle in E. coli. Cell 90: 951-957.

- 1998. Polar localization of the replication origin and terminus in Escherichia coli nucleoids during chromosome partitioning. Genes \& Dev. 12: 1036-1045.

- 1999. Subcellular localization of plasmids containing the oriC region of the Escherichia coli chromosome, with or without the sopABC partitioning system. Mol. Microbiol. 34: 498-503.

Niki, H., A. Jaffé, R. Imamura, T. Ogura, and S. Hiraga. 1991. The new gene mukB codes for a $177 \mathrm{kd}$ protein with coiledcoil domains involved in chromosome partitioning of $E$. coli. EMBO J. 10: 183-193.

Niki, H., R. Imamura, M. Kitaoka, K. Yamanaka, T. Ogura, and S. Hiraga. 1992. E. coli MukB protein involved in chromosome partition forms a homodimer with a rod-and-hinge structure having DNA binding and ATP/GTP binding activities. EMBO J. 11: 5101-5109.

Pettijohn, D.E. 1996. The nucleoid. In Escherichia coli and Salmonella; cellular and molecular biology (ed. F.C. Neidhardt, et al.), pp. 158-166. American Society of Microbiology, Washington, DC.

Pogliano, K., E. Harry, and R. Losick. 1995 Visualization of the subcellular localization of sporulation proteins in Bacillus subtilis using immunofluorescence microscopy. Mol. Microbiol. 18: $459-470$ 
Poulsen, L.K., T.R. Licht, C. Rang, K.A. Krogfelt, and S. Molin. 1995. Physiological state of Escherichia coli BJ4 growing in the large intestines of streptomycin-treated mice. I Bacteriol. 20: $5840-5845$

Rudd, K.E. 1998. Linkage map of Escherichia coli K-12, edition 10: The physical map. Microbiol. Mol. Biol. Rev. 62: 9851019.

Sharpe, M.E., and J. Errington 1996. The Bacillus subtilis sojspoOI locus is required for a centromere-like function involved in prespore chromosome partitioning. Mol. Microbiol. 21: 501-509.

- 1998. A fixed distance for separation of newly replicated copies of oriC in Bacillus subtilis: Implications for co-ordination of chromosome segregation and cell division. Mol. Microbiol. 28: 981-990.

. 1999. Upheaval in the bacterial nucleoid. An active chromosome segregation mechanism. Trends Genet. 15: 7074.

Steiner, W., G. Liu, W.D. Donachie, and P. Kuempel. 1999. The cytoplasmic domain of FtsK protein is required for resolution of chromosome dimers. Mol. Microbiol. 31: 579-583.

Teleman, A.A., P.L. Graumann, D.C.H. Lin, A.D. Grossman, and R. Losick. 1998. Chromosome arrangement within a bacterium. Curr. Biol. 8: 1102-1109.

Wada, M., Y. Kano, T. Ogawa, T. Okazaki, and F. Imamoto. 1988. Construction and characterization of the deletion mutant of hupA and hupB genes in Escherichia coli. J. Mol. Biol. 204: 581-591.

Webb, C.D., A. Teleman, S. Gordon, A. Straight, A. Belmont, D.C.-H. Lin, A.D. Grossman, A. Wright, and R. Losick. 1997. Bipolar localization of the replication origin regions of chromosomes in vegetative and sporulating cells of $B$. subtilis. Cell 88: 667-674.

Webb, C.D., P.L. Graumann, J.A. Kahana, A.A. Teleman, P.A. Silver, and R. Losick. 1998. Use of time-lapse microscopy to visualize rapid movement of the replication origin region of the chromosome during the cell cycle in Bacillus subtilis. Mol. Microbiol. 28: 883-892.

Wu, L.J., and J. Errington. 1998. Use of asymmetric cell division and spoIIIE mutants to probe chromosome orientation and organization in Bacillus subtilis. Mol. Microbiol. 27: 777786.

Yamanaka, K., T. Ogura, H. Niki, and S. Hiraga. 1996. Identification of two new genes, mukE and $m u k F$, involved in chromosome partitioning in Escherichia coli. Mol. \& Gen. Genet. 250: 241-251. 


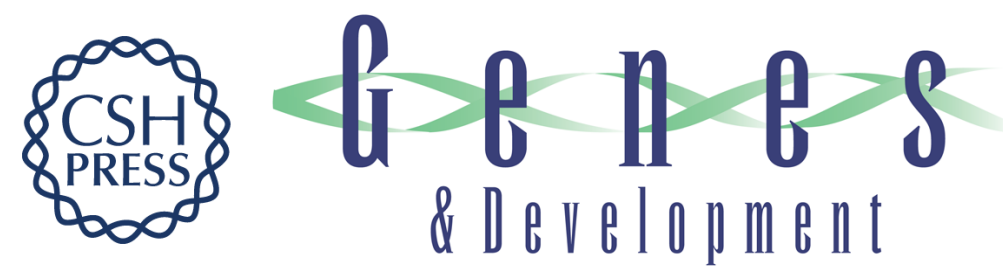

\section{Dynamic organization of chromosomal DNA in Escherichia coli}

Hironori Niki, Yoshiharu Yamaichi and Sota Hiraga

Genes Dev. 2000, 14:

Access the most recent version at doi:10.1101/gad.14.2.212

References This article cites 35 articles, 12 of which can be accessed free at: http://genesdev.cshlp.org/content/14/2/212.full.html\#ref-list-1

License

Email Alerting Receive free email alerts when new articles cite this article - sign up in the box at the top Service right corner of the article or click here.

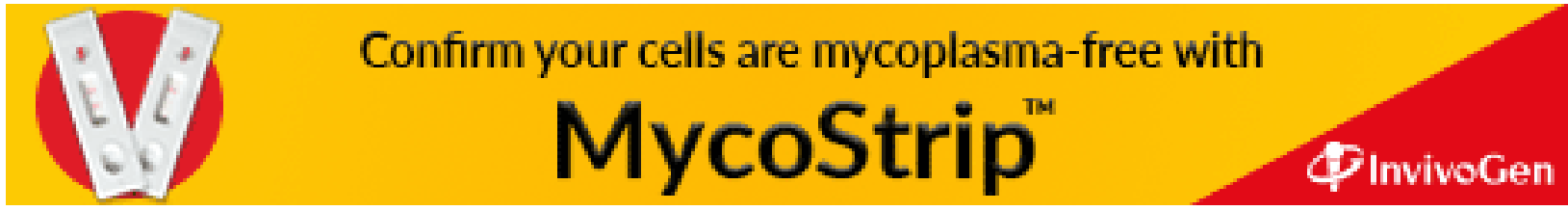

